\title{
Variación Anatómica de la Valva Atrioventricular Derecha
}

\author{
Anatomic Variation of the Right Atrioventricular Valve \\ Oscar Inzunza H.; Waldo Ortega F.; Claudia Berrocal C. \& Alex Vargas D.
}

INZUNZA, H. O.; ORTEGA, F. W.; BERROCAL, C. C. \& VARGAS, D. A. Variación anatómica de la valva atrioventricular derecha. Int. J. Morphol., 24(1):49-52, 2006.

RESUMEN: Si bien la tradición ha consagrado el término "tricúspide" para caracterizar a la valva atrioventricular derecha, numerosos estudios han demostrado la gran variabilidad de presentación de ésta, describiéndose casos con dos y, situaciones extremas, con seis velos valvulares. Parece existir una relación directa entre el incremento del número de válvulas y la morbimortalidad de los portadores de esta variación anatómica. En este trabajo presentamos una valva atrioventricular derecha con dos velos valvulares, estructura donde es posible identificar claramente el componente septal con una sola válvula y el componente mural que se descompone en una extensa válvula anterior y una pequeña válvula comisural anteroseptal.

PALABRAS CLAVE: Valvas cardiacas; Valvas atrioventriculares; Variación anatómica.

\section{INTRODUCCIÓN}

La tradición ha consagrado el término tricúspide para denominar al elemento valvar ubicado en el ostio atrioventricular derecho. Así, este término ya fue utilizado por William Harvey, en su obra señera "De motu cordis", donde describe "la válvula tricúspide está dispuesta, como portero, a la entrada del ventrículo"; señalando allí que, a diferencia de la valva atrioventricular izquierda que él describe "como una mitra", la valva atrioventricular derecha ofrece diferentes presentaciones en los distintos animales, insinuando ya lo vago del término "tricúspide" (Whitteridge, 1964), vocablo que pretende establecer una homología con la superficie coronaria de una pieza dentaria posterior.

Numerosos estudios describen distintas formas de presentación de la valva atrioventricular derecha humana, donde -dependiendo de la serie- los porcentajes de variación oscilan entre el 30\% y el 70\%, observándose valvas con 2, 4, 5, ó 6 cúspides (o válvulas) (Zeren, 1951; Victor \& Nayak, 1994; Sutton et al., 1995; Wafae et al., 1990; Gerola et al., 2001), presentando una menor variabilidad el componente septal de la valva (Gerola et al.).

El análisis funcional comparativo entre las valvas atrioventriculares derecha e izquierda (Victor \& Nayak; Sutton et al.) señala que ambas valvas presentan un componente septal fijo y un componente mural inestable. En la valva atrioventricular derecha, el componente fijo corresponde a la válvula septal, región que permanece relativamente estable durante el ciclo cardiaco; en la valva atrioventricular izquierda esta zona corresponde a la válvula anterior. El componente mural de la valva atrioventricular derecha, aquella zona en relación con el surco atrioventricular y que modifica sus dimensiones durante el ciclo cardiaco, incluye a las válvulas anterior y posterior, presentando -por esta razón- una gran variabilidad; en la valva atrioventricular izquierda, esta zona se corresponde con la válvula posterior, la que presenta, consecuentemente, numerosas hendiduras y festones (Victor \& Nayak; Sutton et al.; Anderson \& Becker 1981).

En este trabajo describimos una variación anatómica de la valva atrioventricular derecha, la cual presenta sólo dos cúspides, válvulas o velos valvulares.

\section{MATERIAL Y MÉTODO}

Se analiza un corazón extraído de un cadáver de sexo masculino, de 62 años de edad, cuyo deceso no se relaciona con una patología cardiovascular, destinado a docencia. El corazón se mantuvo conservado por 2 años, en formalina al $10 \%$. La apertura de éste se realizó con bisturí desde la vena cava superior, a través del atrio derecho, hasta el vértice del ventrículo derecho, siguiendo por el borde lateral o margen agudo de éste 
(Fig. 1). De igual forma, se expuso el lado izquierdo del corazón, desde las venas pulmonares hasta el vértice del ventrículo izquierdo (Fig. 3).

La clasificación de la valva atrioventricular derecha se realizó basándose en el número de válvulas observadas. Los válvulas se definieron como el tejido valvar que queda comprendido entre dos comisuras, según la clasificación tradicional (Silver et al., 1971); y, siguiendo a este autor, las comisuras corresponden a indentaciones profundas de la valva (alcanzan la zona basal de las válvulas), en cuyos bordes libres se insertan cuerdas tendíneas en forma de abanico, provenientes de un músculo papilar.

El corazón fue pesado, procediéndose luego a medir la amplitud y altura de las válvulas con un compás de calibre, colocando especial cuidado en determinar la posición de las válvulas, los músculos papilares y la dirección de sus cuerdas tendíneas.

\section{RESULTADOS}

El corazón pesó $225 \mathrm{~g}$ y, al abrir el atrio derecho mediante un corte entre ambas venas cavas, en el orificio atrioventricular derecho se apreciaban dos válvulas o cúspides, una en relación con la pared septal y otra en relación con la porción mural del ventrículo. Se distinguían dos comisuras: una anterior, donde se ubica un pequeño velo comisural y otra posterior, que carecía de este elemento. La aposición de las válvulas determinaba la formación de una línea de cierre con la forma de una "Y" (Fig. 2).

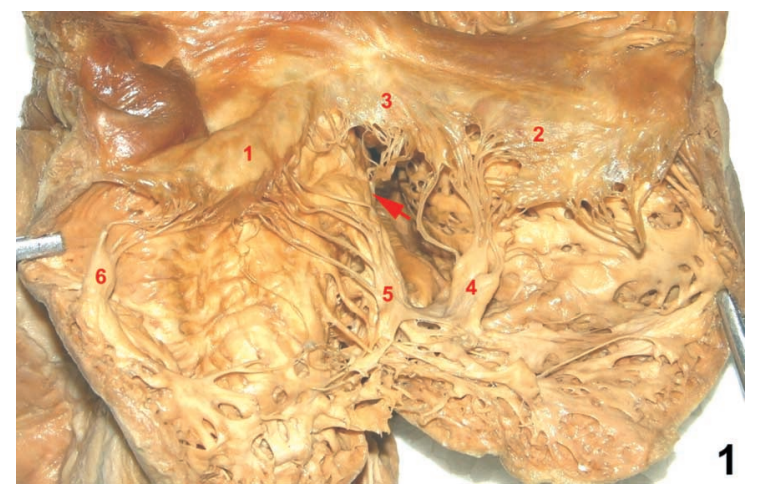

Fig. 1. Ventrículo derecho abierto por el margen agudo del corazón. La válvula atrioventricular derecha está ampliamente extendida para visualizar sus valvas. 1. Válvula septal; 2. Válvula anterior; 3. Válvula comisural anteroseptal; 4. Músculo papilar anterior; 5. Trabécula septomarginal con la inserción de los músculos papilares septales; 8. Músculo papilar posterior; 7. Músculo papilar del cono (flecha). La línea de inserción del componente septal de la valva (1) y del componente mural $(2+3)$, convergen hacia anterior en el borde fijo de la pequeña válvula comisural (3).

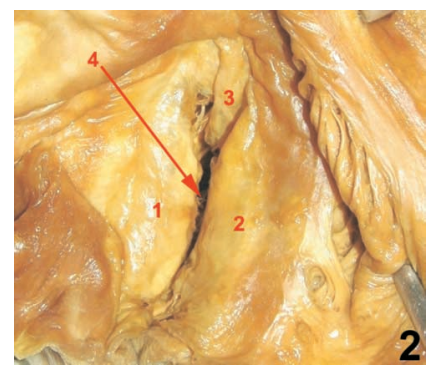

Fig. 2. Valva atrioventricular derecha vista desde el atrio. 1. Válvula septal; 2. Válvula anterior; 3 . Válvula comisural anteroseptal; 4. Línea de aposición de los velos valvulares; 5 . Músculos pectinados. Se observa la línea de aposición con forma de "Y", debido a la presencia de la válvula comisural anteroseptal.

$\mathrm{Al}$ abrir el ventrículo derecho, a través del borde agudo de él, y separar ampliamente sus paredes, hacia medial se observó una válvula septal de forma triangular; opuesta a ella se encontraba la válvula anterior, de forma rectangular; y en la comisura anterior una válvula comisural de forma cuadrangular (Fig. 1).

La válvula septal, triangular, midió $39,8 \mathrm{~mm}$ en su base y $16,1 \mathrm{~mm}$ de altura. Desde el borde anterior de esta válvula surgían cinco cuerdas tendíneas que se insertaban en los músculos papilares septales, dispuestos a lo largo de la trabécula septomarginal. Desde el borde posterior de la válvula se desprendían tres cuerdas tendíneas que se insertaban en el músculo papilar posterior. La válvula anterior, irregularmente rectangular, medía $31,6 \mathrm{~mm}$ en su borde fijo y $15,2 \mathrm{~mm}$ en su parte más alta. Desde la porción anterior de la válvula surgían tres cuerdas tendíneas que se insertaban en el músculo papilar anterior. Así mismo, desde el borde posterior de la válvula se desprendían dos cuerdas tendíneas que se insertaban en el músculo papilar posterior. La válvula comisural anteroseptal, de forma irregularmente cuadrangular, medía $16,3 \mathrm{~mm}$ en su borde fijo y $13,6 \mathrm{~mm}$ en su parte más alta. Desde su porción anterior se desprendían dos cuerdas tendíneas que se insertaban en

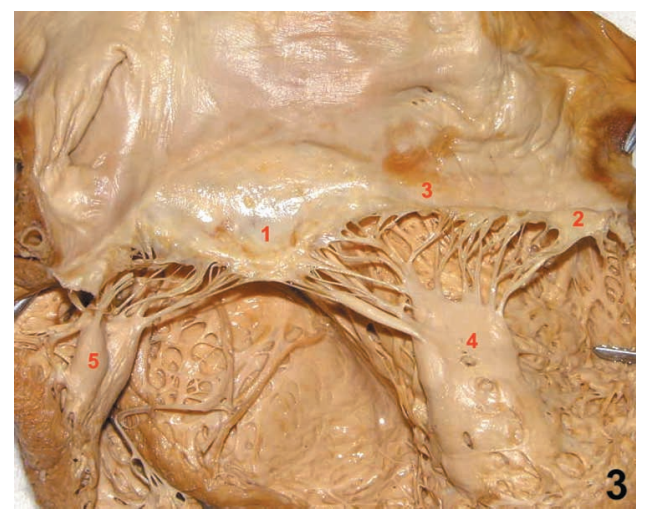

Fig. 3. Ventrículo izquierdo abierto por el margen obtuso del corazón. La valva atrioventricular izquierda está ampliamente extendida para visualizar las válvulas o cúspides. 1. Válvu-

la anterior; 2. Válvula posterior; 3. Festón comisural posteromedial; 4. Músculo papilar posterior; 5. Músculo papilar anterior. Se observa claramente que las cuerdas tendíneas de las válvulas anterior y posterior divergen desde las comisuras valvulares hacia los músculos papilares. El festón comisural posteromedial presenta una cuerda tendínea en forma de abanico que se inserta en el músculo papilar posterior. Se observa que la válvula anterior (aórtica) adopta una forma triangular; y la extensa válvula posterior fue comprometida por el plano de corte a nivel de las cavidades izquierdas, quedando el extremo más anterior de esta válvula sobre el músculo papilar anterior. 


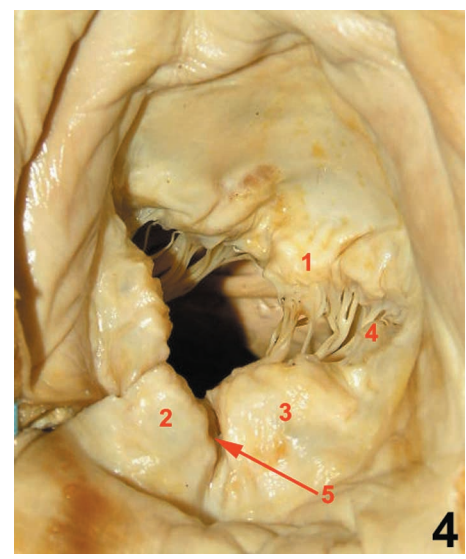

Fig. 4. Valva atrioventricular izquierda vista desde el atrio izquierdo. 1. Válvula anterior; 2. Festón anterior de la válvula posterior; 3 . Festón posterior de la válvula posterior; 4. Festón comisural posteromedial; 5 . Hendidura de la válvula posterior. Se observa que el componente septal de la valva presenta un solo elemento, la válvula anterior; en cambio, el componente mural presenta dos elementos

$(2+3)$ separados por una hendidura. El festón anterior fue comprometido al seccionar las cavidades izquierdas de este corazón.

el músculo papilar del cono (músculo de Lancisi según Anderson et al., 1985) y, desde su porción posterior surgían dos cuerdas tendíneas que se insertaban en el músculo papilar anterior. El músculo papilar anterior presentaba su extremo libre bífido y midió $11,6 \mathrm{~mm}$ de altura. Por su parte, el músculo papilar posterior alcanzaba una altura de $9,1 \mathrm{~mm}$. Por otro lado, los músculos papilares septales correspondían más bien, a engrosamientos de las cuerdas tendíneas, que en número de cinco se iban insertando a lo largo de la trabécula septomarginal, tomando el aspecto de un peine (Fig. 1).

A modo de comparación, en este corazón el ostio atrioventricular izquierdo presentaba una válvula anterior, de forma triangular, cuya base medía $34 \mathrm{~mm}$ y su altura alcanzaba los $20 \mathrm{~mm}$. La válvula posterior, rectangular, medía $50,3 \mathrm{~mm}$ en su borde fijo y presentaba una altura máxima de $13,1 \mathrm{~mm}$; en ella se observó una hendidura que la dividía en un festón anterior de $32,4 \mathrm{~mm}$ y otro posterior de $17,9 \mathrm{~mm}$ de longitud. Esta valva atrioventricular izquierda presenta un pequeño festón comisural posteromedial, de forma cuadrangular, cuyo borde fijo medía 8,6mm y su altura alcanza a 9,1mm. (Figs. 3 y 4).

\section{DISCUSIÓN}

Las variaciones anatómicas constituyen un tema de interés no sólo para quienes cultivamos la morfología (Inzunza et al., 1996a; 1996b) sino también para los clínicos, ya que algunas de estas condiciones, poco frecuentes, pueden asociarse con un aumento de la morbilidad. Sin embargo, un reciente estudio forense, realizado en 400 corazones obtenidos de autopsias, señala que esta variación anatómica - una valva atrioventricular derecha bicúspide-, observada en el 20\% de la muestra, no se relaciona con un aumento de la morbimortalidad; situación que es diametralmente opuesta en aquellos casos que presentan 4 o más válvulas (Kocak et al., 2004).
En la literatura especializada se describe que la disposición de los elementos valvulares del ostio atrioventricular derecho es extremadamente variable (Zeren; Victor \& Nayak; Sutton et al.; Wafae et al.; Gerola et al.), situación que ha llevado a plantear a algunos autores que "la superficie interna del ventrículo derecho es tan particular en cada individuo como lo es la huella digital" (Victor \& Nayak). Estudios filogenéticos comparativos señalan que en los vertebrados con septación interventricular completa, los ostios atrioventriculares derecho e izquierdo presentan un anillo valvar con forma de "D" (Victor et al., 1995), situación que determina la formación de dos unidades funcionales: $1^{\circ}$ La porción recta del anilllo, el componente septal, desde donde se suspende una válvula triangular, elemento que se corresponde con la válvula anterior de la valva atrioventricular izquierda y con la válvula septal de la valva atrioventricular derecha; $y, 2^{\circ}$ la porción curva del anillo, donde se inserta la "gran válvula mural" (Cayré et al., 1993), zona que se corresponde con la válvula posterior de la valva atrioventricular izquierda y con los complejos valvulares anterior y posterior de la valva atrioventricular derecha. Debido a que este componente mural sufre grandes cambios de forma y de tamaño durante el ciclo cardiaco, la "gran válvula mural" en ambos ventrículos debe adaptarse, plegándose, formando así hendiduras y festones, para asegurar un buen cierre valvar. Es interesante citar que en fetos humanos se ha descrito que la valva atrioventricular derecha presenta sólo dos velos valvulares, detectándose en estos especímenes una hendidura en la válvula mural, a nivel del margen agudo del corazón, que la segmenta en un componente anterior y otro posterior (Odgers, 1939). Nuestro hallazgo se corresponde con lo que aquí se ha descrito, de modo que podemos argumentar que desde la porción recta del anillo atrioventricular derecho se extiende la válvula septal, característicamente triangular, y que la "gran válvula mural" presenta dos regiones: Un pequeño segmento anterior, la válvula comisural anteroseptal; $y$, un extenso segmento posterior, la válvula anterior propiamente tal (Fig. 1 y 2). Una situación parecida se aprecia en el anillo atrioventricular izquierdo, donde la porción recta del anillo brinda inserción a la válvula anterior de la valva atrioventricular izquierda y la "gran válvula mural" presenta, en este caso, una hendidura y dos festones (Figs. 3 y 4 ).

Esta división funcional de los ostios atrioventriculares en sus componentes septal y mural parece tener un soporte embriológico, considerando que recientes estudios moleculares y morfogenéticos encuentran una marcada similitud en la formación del componente mural de estas valvas, ya que la válvula posterior de la valva atrioventricular izquierda y las válvulas anterior y posterior de la valva atrioventricular derecha se forman como una protrusión de una lámina del miocardio atrioventricular, con escasa participación de las almohadillas endocárdicas laterales (De Lange et al., 2004). En contraposición, la válvula anterior de la valva atrioventricular izquier- 
da y la válvula septal de la valva atrioventricular derecha se desarrollan a partir del mesénquima de las almohadillas endocárdicas inferior y superior, respectivamente (De la Cruz et al., 1983).

Finalmente, esta homología funcional entre las valvas atrioventriculares izquierda y derecha, con sus componentes septal y mural, no tiene tan sólo un valor académico sino también un interés clínico, siendo posible realizar homoinjertos exitosos, reemplazando una valva atrioventricular derecha dañada por una valva atrioventricular izquierda, respetando, eso sí, la situación de sus componentes funcionales; es decir, rotando la valva atrioventricular izquierda en el sitio receptor, de modo tal que la valva anterior de ella se fija a la porción septal del anillo atrioventricular derecho (Pomar \& Mestres, 1993).

INZUNZA, H. O.; ORTEGA, F. W.; BERROCAL, C. C. \& VARGAS, D. A. Anatomic variation of the right atrioventricular valve. Int. J. Morphol., 24(1):5-6, 2006.

SUMMARY: Anatomic tradition uses the term "tricuspid" for the right atrioventricular valve, even though several studies had demonstrated a great variation in the anatomy of this structure. Some studies describe two valvules (or cusps), others describe six veils. Interestingly, it seems to be a direct relationship between the increment in the number of cusps and the patient morbid-mortality. This study present a right atrioventricular valve bicuspid, whit a clearly septal component with one valvule, and a mural component with a wide anterior valvule and a small anteroseptal commissural cusp.

KEY WORDS: Cardiac valves; Atrioventricular valves; Anatomic variation.

\section{REFERENCIAS BIBLIOGRÁFICAS}

Anderson, R. H. \& Becker, A. Atlas fotográfico de anatomía cardiaca. Barcelona, Ediciones Doyma, 1981.

Anderson, R. H.; Zuberbuhler, J. R.; Penkoske, P. A. \& Neches W. H. Of clefts, commisures and things. J. Thorac. Cardiovac. Surg., 90:605-10, 1985.

Cayré, R.; Valencia-Mayoral, P.; Coffe-Ramírez, V.; Sánchez-Gómez, C.; Angelini, P. \& De la Cruz, M.V. The right atrioventricular valvular apparatus in the chick heart. Acta Anat., 148:27-33, 1993.

De la Cruz, M.V.; Giménez-Ribotta, M.; Saravalli, O. \& Cayré, R. The contribution of the inferior endocardial cushion of the atrioventricular canal to cardiac septation and to the development of the atrioventricular valves: study in the chick embryo. Am. J. Anat., 166 (1):63-72, 1983.

De Lange, F. J.; Moorman, A. F.; Anderson, R. H.; Manner, J.; Soufan, A.T. ; de Gier-de Vries, C.; Schneider, M. D; Webb, S; van den Hoff, M. J. \& Christoffels, V. M. Lineage and morphogenetic analysis of the cardiac valves. Circ Res., 95:645-54, 2004.

Gerola, L. R.; Wafae, N.; Vieira, M. C.; Juliano, Y.; Smith, R. \& Prates, J. C. Anatomic study of the tricuspid valve in children. Surg. Radiol. Anat., 23 (3):149-53, 2001.

Inzunza, O.; Prado, M. \& Valenzuela, A. Variación del origen de la arteria coronaria derecha. Rev. Chil. Cs. Med. Biol., 6 (1):11-4, 1996a.

Inzunza, O.; Valenzuela, A. \& Prado, M. Anastomosis anómala entre las arterias hepática y mesentérica superior. Rev. Chil. Cs. Biol., 6(2):65-8, 1996b.

Kocak, A.; Govsa, F.; Aktas, E. O.; Boydak, B. \& Yavuz, I. C. Structure of the human tricuspid valve leaflets and its chordae tendineae in unexpected death. A forensic autopsy study of 400 cases. Saudi Med. J., 25:1051-9, 2004.
Odgers, P. N. B. The development of the atrioventricular valves in man. J. Anat, 73:643-57, 1939.

Pomar, J. L. \& Mestres, C. A. Tricuspid valve replacement using a mitral homograft: surgical technique and results. J. Heart Valve Dis., 2:125-8, 1993.

Silver, M. D; Lam, J. H. C.; Ranganathan, N. \& Wigle, E. D. Morphology of the human tricuspid valve. Circulation, 43:33349, 1971.

Sutton, J. P. 3rd; Ho, S. Y.; Vogel, M. \& Anderson R. H. Is the morphologically right atrioventricular valve tricuspid? J. Heart Valve Dis., 4 (6):571-5, 1995.

Victor, S. \& Nayak, V. M. The tricuspid valve is bicuspid. J. Heart Valve Dis., 3 (1):27-36, 1994.

Victor, S.; Nayak, V. M.; Raveen, R. \& Gladstone, M. Bicuspid evolution of the arterial and venous atrioventricular valves. $J$. Heart Valve Dis., 4 (1):78-87, 1995.

Wafae, N.; Hayashi, H.; Gerola, L.R. \& Vieira, M.C. Anatomical study of the human tricuspid valve. Surg. Radiol. Anat., 12(1):3741, 1990.

Whitteridge, G. The Anatomical Lectures of William Harvey. Edinburgh. E \& S Livingston Ltd., 1964.

Zeren, Z. Un cas de valvule quinticuspide. Acta Anat., 13:379-84, 1951.

Dirección para correspondencia:

Prof. Dr. Oscar Inzunza $\mathcal{H}$.

Departamento de Anatomía - Facultad de Medicina

Pontificia Universidad Católica de Chile

Casilla 114-D

Santiago - CHILE

Email:oinzunza@puc.cl

Recibido: 12-07-2005

Aceptado:14-12-2005 